\title{
Weaker Functional Pinch Strength Is Associated With Early Thumb Carpometacarpal Osteoarthritis
}

\author{
Thomas J. McQuillan BA, Deborah Kenney MS, OTR, Joseph J. Crisco PhD, \\ Arnold-Peter Weiss MD, Amy L. Ladd MD
}

Received: 24 May 2015/Accepted: 8 October 2015/Published online: 22 October 2015

(C) The Association of Bone and Joint Surgeons (B) 2015

\begin{abstract}
Background The thumb carpometacarpal (CMC) joint orchestrates pinch in its various positions, and thumb CMC osteoarthritis (OA) is a major source of orthopaedic morbidity. Self-reported pain, weakness, and physical examination may not correspond to radiographic findings when diagnosing early thumb CMC OA. Weakness is a
\end{abstract}

One of the authors (TJM) has received research support funding from a Stanford University MedScholars grant. Both institutions of the authors have received, during the study period, funding from the National Institute of Arthritis and Musculoskeletal and Skin Disease of the National Institutes of Health under award number R01AR059185.

All ICMJE Conflict of Interest Forms for authors and Clinical Orthopaedics and Related Research ${ }^{\mathbb{R}}$ editors and board members are on file with the publication and can be viewed on request.

Clinical Orthopaedics and Related Research ${ }^{\mathbb{R}}$ neither advocates nor endorses the use of any treatment, drug, or device. Readers are encouraged to always seek additional information, including FDAapproval status, of any drug or device prior to clinical use.

Each author certifies that his or her institution approved the human protocol for this investigation, that all investigations were conducted in conformity with ethical principles of research, and that informed consent for participation in the study was obtained.

This work was performed at Stanford University, Stanford, CA, USA and Brown University, Providence, RI, USA.

The content of this article is solely the responsibility of the authors and does not necessarily represent the official views of the National Institutes of Health.

T. J. McQuillan ( $₫)$, D. Kenney, A. L. Ladd

Robert A. Chase Hand \& Upper Limb Center, Department of Orthopaedic Surgery, Stanford University, Stanford, CA 94305, USA

e-mail: tmcquill@stanford.edu

J. J. Crisco, A.-P. Weiss

Department of Orthopaedics, Rhode Island Hospital, The Warren

Alpert Medical School of Brown University, Providence, RI,

USA prominent feature of the disease, but little evidence exists to quantify self-reported loss of strength with time, or to compare weakness with that of a nonarthritic population during early disease.

Questions/purposes We asked: (1) Is pinch strength in subjects with early thumb CMC OA less than that in asymptomatic control subjects; and (2) weakness in which pinch position (key, tripod, or tip pinch) has the strongest association with early OA diagnosis?

Methods For this case-control study, we recruited 23 subjects who were asymptomatic and 91 with early OA for comprehensive history, physical examination, strength measurements, pain surveys, and radiographic evaluation. We used multivariate logistic regression to quantify the association between declining pinch strength and early OA diagnosis after controlling for age, sex, and BMI. This analysis was performed for three different pinch positions (key, tripod, and tip pinch) to evaluate which measurement was most closely associated with diagnosis.

Results Pinch strength was less in patients with early thumb CMC OA. Key pinch had the most robust association with OA diagnosis, in which a $20 \%$ decrease in key pinch strength from the control subjects' baseline was associated with a $10 \%$ increase in the OA diagnosis $(95 \%$ $\mathrm{CI}, 3 \%-16 \% ; \mathrm{p}=0.004)$. This had a stronger association with OA diagnosis than tip pinch, in which a $20 \%$ decrease in strength was associated with a $6 \%$ increase in early CMC OA (95\% CI, $1.0 \%-11 \%$; $=0.031)$. Tripod pinch also was associated to a lesser extent; a $20 \%$ reduction in tripod pinch led to a $5 \%$ increase in OA $(95 \% \mathrm{CI}, 1.3 \%-$ $9 \% ; \mathrm{p}=0.048)$.

Conclusions Decreasing pinch strength, especially key pinch, is associated with early CMC arthritis before the development of extensive radiographic disease. 
Clinical Relevance Weakness in pinch strength, especially key pinch, is an important feature in the pathogenesis of early CMC OA and may appear before radiographic disease is present or advanced. These findings suggest a role for intervention in early disease for promoting nonoperative joint protection and strengthening, and designing surgical procedures aimed to delay or prevent clinical and radiographic progression.

\section{Introduction}

Thumb carpometacarpal (CMC) osteoarthritis (OA) is a common degenerative disease associated with significant morbidity and functional impairment. Patients with early CMC OA typically have weakness on the affected hand when pinching, grasping, or twisting objects [26-28]. Studies support an association of advanced age, female sex, family history, and increased BMI with CMC OA [2, 6, 16, 30]. Pinching tasks are especially uncomfortable for patients with early OA, and patients with CMC and distal interphalangeal arthritis have been shown to have lower pinch and grip strength $[1,5,8,17,24]$. The extent to which this weakness during functional tasks informs the diagnosis of early CMC OA has been preliminary but not rigorously quantified in the literature [15, 22]. Halilaj et al. [15] reported that the healthy CMC joint is relatively stable during pinching and twisting, but the joint may have different kinematics in the diseased state. A prior study determined that a strength-dexterity test may be helpful in identifying patients with CMC OA, whereas pure pinch strength did not differ between subjects with OA and normal control subjects [27].

Prior studies of pinch have not controlled for age or focused on early CMC disease, which is a prominent feature of our large cohort. Our study therefore aimed to examine three different modalities of functional pinch strength and their relationship with early thumb CMC OA diagnosis. Specifically, we asked (1) is there lower pinch strength in subjects with early thumb CMC OA compared with asymptomatic control subjects, and (2) which pinch position (key, tripod, or tip pinch) has the strongest association with weakness in early OA diagnosis?

\section{Patients and Methods}

Symptomatic early CMC OA and control subjects were recruited as part of a prospective trial studying the kinematics of the CMC joint. We obtained baseline data on 114 individuals (91 subjects with early OA and 23 controls) who were recruited as part of a larger National Institutes of Health investigation of biomechanics and thumb CMC OA [13-15].
Prior institutional review board approval was obtained at both sites. Radiographs including AP, lateral, stress, and Robert's views of the thumb and a detailed history were used to survey prospective subjects for inclusion and exclusion criteria.

Inclusion of subjects with early OA was defined as pain or discomfort complaints at the base of the thumb and radiographs showing arthrosis graded as modified Eaton [9, 10] Stage 0 or I $[4,7,20]$ (meaning either a normal-appearing joint, or slight arthritic changes including increased subchondral sclerosis or minimal joint narrowing). Exclusion criteria included a history of inflammatory arthritis, connective tissue disease, metabolic bone disease, radiographically evident $\mathrm{OA}$ in other joints of the wrist and trapezium, wrist and thumb fractures, ligamentous injury, and previous ipsilateral hand surgery [12]. Inclusion for control subjects required that they had no complaints of thumb pain and normal (modified Eaton Stage 0) radiographs $[4,7,9,10,20]$.

Self-reported pain, ROM, history of hand use, BeightonHoran score [3], and demographic information were obtained for each subject. Imaging studies included radiographs and $\mathrm{CT}$ for use in other studies.

We measured pinch strength in three tests on a simple pinch meter (Greenleaf Medical Systems, Palo Alto, CA). Pinch positions were defined using the American Society for Hand Therapists guidelines [11, 29]. Key pinch was defined as holding the sensor between the pad of the thumb and the radial aspect of the index finger, located approximately near the proximal interphalangeal joint. Tripod pinch was defined as holding the sensor on the pad of the thumb opposed by the index and long fingers. Tip pinch was defined as holding the sensor on the distal portion of the pads of the thumb and index finger. The maximum force was recorded for each test a total of three times with 45 seconds of recovery allowed between measurements, and these three values were averaged for each test. Key pinch was measured first, followed by tripod pinch, followed by tip pinch, with at least 45 seconds between measurements to explain the hand position for the next test.

The variables in our predictive model included pinch strength, age, sex, and BMI. Pinch strength variables were defined as key pinch, tripod pinch, and tip pinch as described previously. The dependent variable was a binary variable of whether the patient was in the early OA group or the control group. Advanced age, female sex, and increased BMI were previously identified as factors associated with CMC OA, [2, $6,16,30]$ and were controlled for in the adjusted analysis.

\section{Statistical Analysis}

Data were analyzed using Stata ${ }^{\circledR}$ Version 13.1 (StataCorp LP, College Station, TX, USA), generating summary 
statistics for the symptomatic subjects and control subjects. We performed unadjusted regressions and regressions that controlled for age, sex, and BMI to identify the strength and direction of the relationship between pinch strength and the likelihood of OA. To quantify this relationship and develop a clinically useful value, we performed a marginal effects calculation on the adjusted models for key pinch, tripod pinch, and tip pinch. The marginal effects calculation reflects the percentage change in the probability of OA diagnosis for a $1-\mathrm{kg}$ increase in pinch strength after controlling for age, sex, and BMI. The minimum clinically reported difference for grip strength is $19.5 \%$, which we used as a guideline for evaluating differences in pinch strength [17]. After controlling for age, sex, and BMI in the regression, we calculated the increase in likelihood of OA diagnosis if there was a $20 \%$ reduction in pinch strength from the control mean for each different pinch modality.

Summary data were collected for the subjects with early $\mathrm{OA}$ and the asymptomatic control subjects, which revealed that there were no differences in age, sex, and BMI (Table 1). Early OA and control subjects had a mean age of 56 years. There were no differences in the groups in the proportion of males (43\% in control subjects, and $47 \%$ in subjects with OA, $\mathrm{p}=0.749)$ and the control subjects had no difference in average BMI $\left(29 \mathrm{~kg} / \mathrm{m}^{2}\right.$ in control subjects, $27 \mathrm{~kg} / \mathrm{m}^{2}$ in symptomatic subjects, $\mathrm{p}=0.172$ ).

\section{Results}

All three means of pinch strength (key pinch, tripod pinch, tip pinch) were lower in the early OA group than in the control group before controlling for age, sex, and BMI (Table 2). Only key pinch strength differed between the groups before adjusting for known risk factors. After adjusting for age, sex, and BMI, all three types of pinch were associated with early OA diagnosis (Table 3). Strength measurements for pinch were greatest for the key pinch position, followed by tripod pinch, and finally the tip pinch, which held true regardless of sex. For key pinch, OA was associated with less strength (adjusted log odds ratio, $0.41 ; 95 \%$ CI, 0.13-0.69; $\mathrm{p}=0.004)$. A $20 \%$ reduction in key pinch corresponded to a $10 \%$ (95\% CI, 3\%-16\%) increase in likelihood of early CMC OA. A $20 \%$ reduction in tripod pinch was associated with a 5\% (95\% CI, 1\%-9\%; $\mathrm{p}=0.048)$ increase in OA diagnosis and a $6 \%(95 \% \mathrm{CI}$, $1 \%-11 \% ; \mathrm{p}=0.031)$ increase in likelihood for tip pinch (Table 3).

Key pinch strength was the most robust indicator of early OA diagnosis. Marginal effects calculation of the logistic regression showed that every $1-\mathrm{kg}$ decrease in key pinch strength was associated with a $6 \%$ increase in the likelihood of OA diagnosis (95\% CI, 2\%-10\%; $\mathrm{p}=0.004)$. For perspective, the average key pinch strength for the healthy control subjects was approximately $8 \mathrm{~kg}$ with a SD of $2 \mathrm{~kg}$ (Table 2). Therefore, a decrease of $1 \mathrm{~kg}$ in pinch strength, which corresponds to an approximately $12 \%$ loss of strength compared with the control mean, was associated with a $6 \%$ increased likelihood of OA diagnosis independent of other predictors like advanced age, female sex, and increased BMI. Key pinch represented a strong clinical predictor of early CMC OA diagnosis in the presence of these other risk factors.

A $1-\mathrm{kg}$ decrease in tripod pinch was associated with a $4 \%$ decrease in the likelihood of OA $(95 \%$ CI, $0.02 \%-8 \%$; $\mathrm{p}=0.048$ ). We noted that the average tripod pinch among control subjects was $6 \mathrm{~kg}$ with a SD of $2 \mathrm{~kg}$; a 1-kg loss of strength would correspond to a $16 \%$ reduction from the mean. A 1-kg decrease in tip pinch strength was associated with a $6 \%$ increase in the likelihood of OA diagnosis $(95 \%$

Table 1. Summary statistics

\begin{tabular}{lllr}
\hline Variable & $\begin{array}{l}\text { Control subjects, } \\
\text { mean }(\mathrm{SD}) \mathrm{n}=23\end{array}$ & $\begin{array}{l}\text { Subjects with osteoarthritis, } \\
\text { mean (SD) } \mathrm{n}=91\end{array}$ & $\mathrm{p}$ value \\
\hline Age (years) & $56.1(17.8)$ & $56.4(7.6)$ & 0.902 \\
Male sex & $0.4(0.5)$ & $0.5(0.5)$ & 0.749 \\
BMI $\left(\mathrm{kg} / \mathrm{m}^{2}\right)$ & $28.6(6.0)$ & $26.9(5.2)$ & 0.172 \\
\hline
\end{tabular}

Table 2. Baseline pinch strength

\begin{tabular}{lllr}
\hline Variable & $\begin{array}{l}\text { Control subjects, } \\
\text { mean }(\mathrm{SD}) \mathrm{n}=23\end{array}$ & $\begin{array}{l}\text { Subjects with osteoarthritis, } \\
\text { mean }(\mathrm{SD}) \mathrm{n}=91\end{array}$ & $\mathrm{p}$ value \\
\hline Key pinch strength $(\mathrm{kg})$ & $8.11(1.95)$ & $7.00(2.26)$ & 0.033 \\
Tripod pinch strength $(\mathrm{kg})$ & $6.43(2.27)$ & $5.69(2.08)$ & 0.139 \\
Tip pinch strength $(\mathrm{kg})$ & $4.72(1.56)$ & $4.21(1.41)$ & 0.131 \\
\hline
\end{tabular}


Table 3. Effect of pinch strength on the probability of OA diagnosis

\begin{tabular}{|c|c|c|c|c|}
\hline Variable & Adjusted log odds ratio & $\begin{array}{l}\text { Increase in OA diagnosis } \\
\text { per } \mathrm{kg} \text { decreased pinch }\end{array}$ & $\begin{array}{l}\text { Increase in OA diagnosis with } \\
20 \% \text { decreased pinch }\end{array}$ & $\mathrm{p}$ value \\
\hline Key pinch & $0.41(0.13-0.69)$ & $0.06(0.02-0.10)$ & $9.7 \%(3.3 \%-16.2 \%)$ & 0.004 \\
\hline Tripod pinch & $0.17(0.00-0.50)$ & $0.04(0.01-0.07)$ & $4.9 \%(1.3 \%-9.0 \%)$ & 0.048 \\
\hline Tip pinch & $0.41(0.03-0.79)$ & $0.06(0.01-0.12)$ & $6.0 \%(1.0 \%-11.4 \%)$ & 0.031 \\
\hline
\end{tabular}

$\mathrm{OA}=$ osteoarthritis; 95\% CI in parentheses.

CI, $1 \%-12 \% ; \mathrm{p}=0.031)$ when controlling for other risk factors. The mean tip pinch strength was $5 \mathrm{~kg}$ with an SD of $2 \mathrm{~kg}$, which was lower than the other risk factors; a 1-kg decrease represents a proportionally greater loss in strength.

\section{Discussion}

The purpose of this study was to evaluate three types of pinch as an indicator of early CMC OA. To isolate the association between decreased pinch strength and early OA diagnosis, we controlled for age, sex, and BMI, which have been reported as risk factors for developing early CMC OA $[2,25]$. Prior studies have not controlled for the effect of known risk factors for $\mathrm{CMC} \mathrm{OA}$, and have only noted anecdotally that weakness and instability is a feature of CMC disease [15, 22, 27]. Our study was the first of its kind, to our knowledge, to show a decrease in pinch strength in subjects with early CMC arthrosis (Eaton Stage 0 or I) compared with healthy control subjects after controlling for associated risk factors. These findings were particularly prominent when looking at key pinch in our subjects with early CMC OA. This decrease in strength supports the notion that the pathogenesis of CMC OA involves substantial weakness at the joint before prominent radiographic changes (Eaton Stages III and IV).

Decreased key pinch had the strongest association with CMC OA, as a $20 \%$ loss in strength was associated with approximately a $10 \%$ increase in likelihood of diagnosis. This confirmed our initial hypothesis; clinically, patients with CMC OA often present with limitations such as turning keys in doorknobs or manipulating fine objects [23]. Prior research also has implied that the key pinch position is associated with translation of the trapezium and instability at the CMC joint [15], specific cartilage wear patterns [18, 21], and increased mechanical load on the trapezium [19]. This theory supports the notion that the kinematics of the CMC joint during key pinch may be most affected by early weakness and instability.

Several study limitations deserve mention. Strength testing cannot replace history, physical examination, and radiographic findings in the diagnosis and treatment of
CMC OA. No sign or symptom is pathognomonic for OA, and the interpretation of quantitative measurements at baseline is difficult owing to the variability of strength in the population. The diminished strength at baseline in our OA population is important to quantify but cannot be extrapolated for individual patients without observing a trend in their individual strength measurements.

This study supports obtaining a careful history of pinch weakness and instability in patients presenting with CMC symptoms, even in the absence of radiographic disease. This cohort of symptomatic subjects with early OA will be assessed longitudinally with followup imaging, strength measurements, and surveys of pain and function to further analyze the biomechanics and natural history of thumb CMC OA.

Acknowledgments We also thank Tarpit Patel MS and Eni Halilaj $\mathrm{PhD}$ from the Bioengineering Laboratory, Department of Orthopaedics, at Brown University (Providence, RI, USA) for their invaluable contributions to our work.

\section{References}

1. Bagis S, Sahin G, Yapici Y, Cimen OB, Erdogan C. The effect of hand osteoarthritis on grip and pinch strength and hand function in postmenopausal women. Clin Rheumatol. 2003;22:420-424.

2. Becker SJ, Briet JP, Hageman MG, Ring D. Death, taxes, and trapeziometacarpal arthrosis. Clin Orthop Relat Res. 2013;471:37383744.

3. Beighton P, Horan F. Orthopaedic aspects of the Ehlers-Danlos syndrome. J Bone Joint Surg Br. 1969;51:444-453.

4. Berger AJ, Momeni A, Ladd AL. Intra- and interobserver reliability of the Eaton classification for trapeziometacarpal arthritis: a systematic review. Clin Orthop Relat Res. 2014;472:1155-1159.

5. Cha SM, Shin HD, Kim KC, Park JW. Comparison of grip strength among 6 grip methods. J Hand Surg Am. 2014;39:22772284.

6. Dahaghin S, Bierma-Zeinstra SM, Koes BW, Hazes JM, Pols HA. Do metabolic factors add to the effect of overweight on hand osteoarthritis? The Rotterdam Study. Ann Rheum Dis. 2007;66:916-920.

7. Dela Rosa TL, Vance MC, Stern PJ. Radiographic optimization of the Eaton classification. J Hand Surg Br. 2004;29:173-177.

8. Dominick KL, Jordan JM, Renner JB, Kraus VB. Relationship of radiographic and clinical variables to pinch and grip strength among individuals with osteoarthritis. Arthritis Rheum. 2005;52:1424-1430.

9. Eaton RG, Glickel SZ. Trapeziometacarpal osteoarthritis: staging as a rationale for treatment. Hand Clin. 1987;3:455-471. 
10. Eaton RG, Littler JW. Ligament reconstruction for the painful thumb carpometacarpal joint. J Bone Joint Surg Am. 1973;55:1655-1666.

11. Fess E, Moran C. Clinical Assessment Recommendations. Garner, NC: American Society for Hand Therapists; 1981.

12. Gamble JG, Mochizuki C, Rinsky LA. Trapeziometacarpal abnormalities in Ehlers-Danlos syndrome. J Hand Surg Am. 1989;14:89-94.

13. Halilaj E, Laidlaw DH, Moore DC, Crisco JJ. How do sex, age, and osteoarthritis affect cartilage thickness at the thumb carpometacarpal joint? Insights from subject-specific cartilage modeling. In: Tavares JM, Luo X, Li S, eds. Bio-Imaging and Visualization for Patient-Customized Simulations. Vol 13. Cham, Switzerland: Springer International Publishing; 2014:103-111.

14. Halilaj E, Moore DC, Laidlaw DH, Got CJ, Weiss AP, Ladd AL, Crisco JJ. The morphology of the thumb carpometacarpal joint does not differ between men and women, but changes with aging and early osteoarthritis. J Biomech. 2014;47:2709-2714.

15. Halilaj E, Rainbow MJ, Got C, Schwartz JB, Moore DC, Weiss AP, Ladd AL, Crisco JJ. In vivo kinematics of the thumb carpometacarpal joint during three isometric functional tasks. Clin Orthop Relat Res. 2014;472:1114-1122.

16. Hunter DJ, Zhang Y, Sokolove J, Niu J, Aliabadi P, Felson DT. Trapeziometacarpal subluxation predisposes to incident trapeziometacarpal osteoarthritis (OA): the Framingham Study. Osteoarthritis Cartilage. 2005;13:953-957.

17. Kim JK, Park MG, Shin SJ. What is the minimum clinically important difference in grip strength? Clin Orthop Relat Res. 2014;472:2536-2541.

18. Koff MF, Ugwonali OF, Strauch RJ, Rosenwasser MP, Ateshian GA, Mow VC. Sequential wear patterns of the articular cartilage of the thumb carpometacarpal joint in osteoarthritis. J Hand Surg Am. 2003;28:597-604.

19. Kovler M, Lundon K, McKee N, Agur A. The human first carpometacarpal joint: osteoarthritic degeneration and 3-dimensional modeling. J Hand Ther. 2004;17:393-400.
20. Ladd AL, Messana JM, Berger AJ, Weiss AP. Correlation of clinical disease severity to radiographic thumb osteoarthritis index. J Hand Surg Am. 2015;40:474-482.

21. Lee AT, Williams AA, Lee J, Cheng R, Lindsey DP, Ladd AL. Trapezium trabecular morphology in carpometacarpal arthritis. $J$ Hand Surg Am. 2013;38:309-315.

22. Luker KR, Aguinaldo A, Kenney D, Cahill-Rowley K, Ladd AL. Functional task kinematics of the thumb carpometacarpal joint. Clin Orthop Relat Res. 2014;472:1123-1129.

23. Marshall M, van der Windt D, Nicholls E, Myers H, Hay E, Dziedzic K. Radiographic hand osteoarthritis: patterns and associations with hand pain and function in a communitydwelling sample. Osteoarthritis Cartilage. 2009;17:1440-1447.

24. Mathiowetz V, Kashman N, Volland G, Weber K, Dowe M, Rogers S. Grip and pinch strength: normative data for adults. Arch Phys Med Rehabil. 1985;66:69-74.

25. Sonne-Holm S, Jacobsen S. Osteoarthritis of the first carpometacarpal joint: a study of radiology and clinical epidemiology. Results from the Copenhagen Osteoarthritis Study. Osteoarthritis Cartilage. 2006;14:496-500.

26. Tsai P, Beredjiklian PK. Physical diagnosis and radiographic examination of the thumb. Hand Clin. 2008;24:231-237, v.

27. Valero-Cuevas FJ, Smaby N, Venkadesan M, Peterson M, Wright $\mathrm{T}$. The strength-dexterity test as a measure of dynamic pinch performance. J Biomech. 2003;36:265-270.

28. Van Heest AE, Kallemeier P. Thumb carpal metacarpal arthritis. J Am Acad Orthop Surg. 2008;16:140-151.

29. Villafañe JH, Valdes K. Reliability of pinch strength testing in elderly subjects with unilateral thumb carpometacarpal osteoarthritis. J Phys Ther Sci. 2014;26:993-995.

30. Wolf JM, Scher DL, Etchill EW, Scott F, Williams AE, Delaronde S, King KB. Relationship of relaxin hormone and thumb carpometacarpal joint arthritis. Clin Orthop Relat Res. 2014;472:1130-1137. 